\title{
INVERTING THE POSITION-VELOCITY DIAGRAMS OF MOLECULAR DISCS
}

\author{
J.S. Richer and R. Padman \\ Mullard Radio Astronomy Observatory Cambridge, England
}

The molecular discs around Young Stellar Objects can be detected through high spatial and spectral resolution observations of their position-velocity diagrams. By mapping a high lying transition of a density-tracing molecule (e.g. $\mathrm{HCN}, \mathrm{HCO}^{+}, \mathrm{NH}_{3}$ ), the lines should be optically thin and we can expect to see the signature of rotation of the protostellar accretion disc. We present single-dish observations of the S106 system which show evidence for the presence of such a rotating disc. We also discuss a simple disc model which predicts the form of the resulting $1-v$ diagram. With high quality data, we should be able to do the inverse problem that of recovering the disc properties from the l-v diagram. We discuss approaches to this inverse problem both by a Singular Value Decomposition (least-squares) method, and by a Maximum Entropy Method.

\section{IDENTIFICATION OF OUTFLOW EXCITING SOURCES THROUGH AMMONIA OBSERVATIONS}

\author{
G. Anglada ${ }^{1,2}$, L.F. Rodriguez ${ }^{3,4}$, J. M. Torrelles ${ }^{5}$, R. Estalella ${ }^{1,2}$, P.T.P. \\ $\mathrm{Ho}^{3}$, J. Canto ${ }^{6}$, R. López ${ }^{1,2}$, and L. Verdes-Montenegro ${ }^{5}$ \\ ${ }^{1}$ Department de Física de l' Atmósfera, Universitat de Barcelona \\ ${ }^{2}$ Grup d'Astrofísica, Societat Catalana de Física, IEC \\ ${ }^{3}$ Harvard-Smithsonian Center for Astrophysics \\ ${ }^{4}$ On sabbatical leave from Instituto de Astronomía, UNAM \\ ${ }^{5}$ Instituto de Astrofísica de Andalucía, CSIC \\ ${ }^{6}$ Instituto de Astronomía, UNAM
}

Using the 37-m telescope of the Haystack Observatory, we observed the $(\mathrm{J}, \mathrm{K})=(1,1)$ ammonia transition towards the suspected exciting sources of twelve regions with molecular or optical outflows: L1448, L1455, L1524 (Haro 6-10), RNO 43, HH 34, HH 38,43, Haro 4-255 FIR, NGC 2264 (HH 14-4,6), L43, R CrA, HH 32a (AS353A), and V1331 Cyg. We detected and mapped ammonia emission in nine of these regions.

In five of mapped regions, the spatial coincidence of the high-density gas with the objects previously proposed as exciting sources gives support to those identifications. For L1524 (Haro 6-10) and HH 38,43 we propose a new location for their exciting sources. We detected a radio continuum source and an unusually strong $\mathrm{H}_{2} \mathrm{O}$ maser coinciding with the maximum of the ammonia emission in L1448. This region appears to be the site of very recent star formation. 